\title{
Significance of Preoperative Peripheral Blood Neutrophil-lymphocyte Ratio in Predicting Postoperative Survival in Patients with Multiple Myeloma Bone Disease
}

\author{
Ziyu Xu \\ Beijing Chaoyang Hospital \\ Xingchen Yao \\ Beijing Chaoyang Hospital \\ Xiangjun Shi \\ Beijing Chaoyang Hospital \\ xinru du ( $\sim$ dxr2013@163.com ) \\ Beijing Chaoyang Hospital
}

Research

Keywords: multiple myeloma bone disease (MMBD), poorer prognosis and shorter POS

Posted Date: December 30th, 2020

DOl: https://doi.org/10.21203/rs.3.rs-136507/v1

License: (c) (i) This work is licensed under a Creative Commons Attribution 4.0 International License.

Read Full License 


\section{Abstract}

Background: By observing the change characteristics of NLR at different stages after operation, to make a preliminary analysis of the effect of operation on the immune status of MM patients and study the prognostic value of peripheral blood NLR in predicting the treatment of multiple myeloma bone disease (MMBD).

Method: The clinical data of 82 MMBD patients who underwent operations in our hospital were collected, which to analyze the effect of preoperative peripheral blood NLR on the prognosis of MMBD patients; Peripheral blood lymphocyte percentage was taken as the major observation indicator to analyze the change characteristics of the immune status of MM patients.

Results: Data showed that the NLR cut-off values of the NLR $\geq 3$ group and NLR $\geq 4$ group were significantly correlated with POS. The POS of NLR $\geq 3$ patients (14.86 \pm 14.28$)$ was significantly shorter than that of NLR $₫ 3$ patients $(32.68 \pm 21.76)$. The lymphocyte percentage 1 week after operation $(19.329 \pm 9.083)$ was significantly lower than that before operation $(25.723 \pm 11.016)$. Survival analysis showed that postoperative chemotherapy and preoperative peripheral blood NLR were independent risk factors for POS.

Conclusions: NLR was significant for the prediction of the POS of MMBD patients treated with operation, and MMBD patients with high preoperative NLR $(N L R \geq 3)$ had a poorer prognosis and shorter POS. The immune status of MMBD patients was at a normal low level, and significantly declined after operation.

Trial registration: As this was a retrospective study, it did not require ethical approval; all patients had signed informed consent when they received treatment, and all treatment options were voluntary.

\section{Introduction}

Multiple myeloma (MM) is a malignant proliferative disease of plasma cells, having the second incidence among blood tumor diseases. With the growth of the aging population, the incidence of MM increases, and there are many studies on prognosis, especially on prognostic factors and relevant stratification data. Such studies are based on the characteristics of patients, tumor load and cytogenetics ${ }^{[1]}$, and mainly target the selection of chemotherapy regimens and other medical treatments. However, there is little literature on prognosis stratification of MM surgical treatment ${ }^{[2-4]}$.

As a marker of systematic inflammation, neutrophil-lymphocyte ratio (NLR) has been used in the diagnosis of infectious diseases. In recent years, some scholars used it as an immune status evaluation indicator to assess the prognosis of solid tumors, such as hepatocellular carcinoma, gastric cancer, renal cell carcinoma, colorectal cancer, ovarian cancer and prostate cancer. Their studies suggested that a high NLR predicted a poor prognosis in patients ${ }^{[5-7]}$, while there were few studies on the role of NLR in hematological diseases. Some studies have shown that elevated NLR may lead to shorter overall survival of MM patients, and a high NLR is an independent risk factor for the progression-free survival of 
patients ${ }^{[8]}$. Besides, immune injuries may also be an important factor affecting the prognosis of MM according to some scholars ${ }^{[9]}$. However, there are no reports on whether there is any change in the immune status of MM patients before and after operation and whether NLR can be used for the prognostic evaluation of multiple myeloma bone disease (MMBD) treated surgically.

The study intends to analyze the value of preoperative peripheral blood NLR in predicting patients' prognosis and its effect on PS by reviewing the general condition, indicators, operation information and follow-up visits of MMBD patients that were admitted to the hospital and underwent operations from 2007 to 2017, and to study preliminarily the effect of operations on the immune status of patients with reference to the changes of absolute neutrophil count and absolute lymphocyte absolute at different time points and the change of lymphocyte percentage before and after operation.

\section{Materials And Methods}

\section{General data}

MMBD patients that underwent operations at the hospital from 2007 to 2017 and did not suffer coronary heart disease, chronic lung disease, severe infection or other hematological diseases significantly affecting the peripheral blood neutrophil count or peripheral blood lymphocyte count, as well as other patients participating in clinical drug trials, were recruited, totaling 82 patients. The sites and number of their lesions were determined according to CT and MRI findings. General information of patients, including their age, gender, DS stage, ISS stage, type, lesion site, preoperative/postoperative treatment, operation scheme and stem cell transplantation therapy, were collected. Their NLRs were calculated according to their peripheral blood absolute neutrophil counts, absolute lymphocyte counts and lymphocyte percentages before operation, 1 week and 1 month after operation and at the last follow-up visit. And their cumulative survival rates were calculated according to their survival 1 year, 3 years and 5 years after operation.

\section{Statistical analysis}

Kaplan-Meier method was used to draw the patients' survival curves with different preoperative peripheral blood NLRs, so as to analyze the correlation between preoperative peripheral blood NLR and postoperative survival (POS). Chi-square test was used to analyze the 1-year, 3-year and 5-year cumulative survival rates of patients with different preoperative peripheral blood NLRs, so as to calculate the cut-off value, select the NLR cut-off value with the most significant difference for grouping, and perform a univariate analysis on clinical data that might affect the NLR cut-off value. Peripheral blood lymphocyte percentage was used as the evaluation indicator to assess preliminarily the immune status of $82 \mathrm{MMBD}$ patients before and after operation, so as to perform a univariate analysis on the lymphocyte percentages of the 82 postoperative patients at different stages and preliminarily assess the effect of operations on the immune status of MMBD patients. A multivariate analysis was performed on the clinical data that might affect the POS of MMBD patients. All data were analyzed in SPSS 19.0 
statistical software, with the measurement data expressed in the form of mean \pm standard deviation. The difference when $P<0.05$ was deemed to have statistical significance.

\section{Results}

Among the 82 MMBD patients that underwent operative treatment, 44 were male $(53.7 \%)$ and 38 were female (46.3\%), with an average age of $60.33 \pm 9.62$ years old. Subtypes of MM: 45 of the patients (54.9\%) were of IgG subtype, 23 (28\%) were of IgA subtype, 3 (3.7\%) were of IgD subtype, and 11 (13.4\%) were of light-chain $\mathrm{k} / \lambda$ subtype. $15(18.3 \%)$ patients were at ISS Stage I, $37(45.1 \%)$ were at ISS Stage II, and $30(36.6 \%)$ were at ISS Stage III. Lesion site: 56 patients $(68.3 \%)$ had their lesions at the spine, 19 $(23.2 \%)$ at the long bone of the limb, and 7 at the soft tissue $(8.5 \%)$.

\section{Treatment Status}

Before operation, 48 patients (58.5\%) received chemotherapy, while 34 (41.5\%) received none. After operation, 71 patients $(86.6 \%)$ received chemotherapy, while $11(13.4 \%)$ received none. 9 patients (11\%) underwent complete excision, $72(87.8 \%)$ underwent partial excision/intra-lesion curettage, and 1 (1.2\%) underwent tissue biopsy. 20 patients $(24.4 \%)$ received stem cell transplantation, while $62(75.6 \%)$ received none. The average POS was $27.03 \pm 21.31$ months (Table 1). 
Table 1

General Information of the 82 MMBD Patients that Underwent Operations

\begin{tabular}{|ll|}
\hline Characteristic & Value N (\%) or SD[] \\
\hline Age & $60.33[9.620]$ \\
\hline Gender & \\
\hline Male & $44(53.7 \%)$ \\
\hline ISS stage & $38(46.3 \%)$ \\
\hline ISS-I & $15(18.3 \%)$ \\
\hline ISS-II & $37(45.1 \%)$ \\
\hline ISS-III & $30(36.6 \%)$ \\
\hline Number of lesions & \\
\hline 1 & $53(64.6 \%)$ \\
\hline 2 & $18(22.0 \%)$ \\
\hline$\geq 3$ & $11(13.4 \%)$ \\
\hline Site of lesion & \\
\hline Spine & $56(68.3 \%)$ \\
\hline Long bone of limb & $19(23.2 \%)$ \\
\hline Soft tissue & $7(8.5 \%)$ \\
\hline Type & $34(41.5 \%)$ \\
\hline IgA & $23(28 \%)$ \\
\hline IgD & $3(5.7 \%)$ \\
\hline IgG & $11(13.4 \%)$ \\
\hline Light chain K/ & \\
\hline Preoperative chemotherapy & \\
\hline Yes & \\
\hline No & \\
\hline Postoperative chemotherapy & \\
\hline
\end{tabular}




\begin{tabular}{|ll|}
\hline Characteristic & Value N (\%) or SD [] \\
\hline No & $11(13.4 \%)$ \\
\hline Operation scheme & \\
\hline Complete excision & $9(11 \%)$ \\
\hline Partial excision/intramedullary curettage & $72(87.8 \%)$ \\
\hline Tissue biopsy & $1(1.2 \%)$ \\
\hline Stem cell transplantation & \\
\hline Yes & $20(24.4 \%)$ \\
\hline No & $62(75.6 \%)$ \\
\hline POS & $27.03 \pm 21.31$ \\
\hline Total survival & $46.63 \pm 31.04$ \\
\hline
\end{tabular}

\section{Effect of preoperative peripheral blood NLR on the POS of MMBD patients}

After analyzing statistically the correlation between preoperative peripheral blood NLR and POS, it was observed that the NLR cut-off values of the preoperative peripheral blood NLR $\geq 3$ group and the preoperative peripheral blood NLR $\geq 4$ group were significantly correlated with the POS and postoperative cumulative survival rate. In particular, when the NLR cut-off value was set as 3 , the difference was the most significant, that is, the POS of NLR $\geq 3$ patients $(n=26)(14.86 \pm 14.28$ months $)$ was significantly lower than that of NLR囚3 patients $(n=56)(32.68 \pm 21.76$ months $)(P<0.001)$ (Table 2) (Fig. 1-4).

Table 2

Comparison of 1-year, 3-year and 5-year Cumulative Survival Rates between the High NLR Group and the Low NLR Group

\begin{tabular}{|c|c|c|c|c|c|}
\hline $\begin{array}{l}\text { Cut-off value of } \\
\text { NLR }\end{array}$ & 1-yr PS* & 3-yr PS* & 5-yr PS* & $\begin{array}{l}\text { Chi- } \\
\text { square }\end{array}$ & $\begin{array}{l}\mathrm{P} \\
\text { Value }\end{array}$ \\
\hline$N L R \geq 4$ & $\begin{array}{l}41.7 \% \text { vs. } \\
82.5 \%\end{array}$ & $\begin{array}{l}10.4 \% \text { vs. } \\
60.9 \%\end{array}$ & $0.0 \%$ vs. $41.7 \%$ & 15.969 & $0.000 *$ \\
\hline$N L R \geq 3(n=26)$ & $\begin{array}{l}52.0 \% \text { vs. } \\
87.3 \%\end{array}$ & $\begin{array}{l}19.1 \% \text { vs. } \\
67.2 \%\end{array}$ & $0.0 \%$ vs. $48.3 \%$ & 22.192 & $0.000 *$ \\
\hline$N L R \geq 2$ & $\begin{array}{l}65.8 \% \text { vs. } \\
88.9 \%\end{array}$ & $\begin{array}{l}46.5 \% \text { vs. } \\
61.1 \%\end{array}$ & $\begin{array}{l}31.0 \% \text { vs. } \\
34.0 \%\end{array}$ & 2.015 & 0.156 \\
\hline $\mathrm{NLR} \geq 1$ & $\begin{array}{l}75.3 \% \text { vs. } \\
85.7 \%\end{array}$ & $52.6 \%$ vs. - & $30.6 \%$ vs. - & 0.459 & 0.498 \\
\hline Note: ${ }^{*} P<0.05$ & & & & & \\
\hline
\end{tabular}




\section{General Condition Of Patients On Preoperative Peripheral Blood NIr}

Patients were grouped into a high NLR group (NLR $\geq 3$ ) and a low NLR group (NLR囚3) according to their NLR values. Univariate analysis of them showed that the number of MMBD lesions affected NLR to some extent $(P=0.033)$, while gender, ISS stage, lesion sites, chemotherapy and stem cell transplantation did not affect the NLR. Preoperative $\mathrm{Hb}$ and AST had differences between the high NLR group and the low NLR group ( $p=0.047, p=0.007$ ), while PLT, ALb, ALT, $\Gamma-G T$, blood $\beta 2-M G$ and $C r$ had no significant statistical difference between the two groups (Table 3). 
Table 3

Univariate Analysis of the General Information of Patients in the High NLR Group and the Low NLR Group

\begin{tabular}{|c|c|c|c|}
\hline Characteristic & NLR囚3(n = 56) & $N L R \geq 3(n=26)$ & P Value \\
\hline \multicolumn{4}{|c|}{ General background information } \\
\hline Age & $60.93 \pm 9.875$ & $59.04 \pm 9.097$ & 0.411 \\
\hline Gender & & & 0.981 \\
\hline Male & $30(53.6 \%)$ & $14(53.8 \%)$ & \\
\hline Female & $26(46.4 \%)$ & $12(46.2 \%)$ & \\
\hline ISS stage & & & 0.693 \\
\hline ISS-I & $10(17.9 \%)$ & $5(19.2 \%)$ & \\
\hline ISS-II & 27 (48.2\%) & $10(38.5 \%)$ & \\
\hline ISS-III & $19(33.9 \%)$ & $11(42.3 \%)$ & \\
\hline Number of lesions & & & $0.033^{*}$ \\
\hline 1 & $41(73.2 \%)$ & $12(46.2 \%)$ & \\
\hline 2 & $8(14.3 \%)$ & $10(38.5 \%)$ & \\
\hline$\geq 3$ & $7(12.5 \%)$ & $4(15.4 \%)$ & \\
\hline Site of lesion & & & 0.982 \\
\hline Spine & $38(67.9 \%)$ & $18(69.2 \%)$ & \\
\hline Long bone of limb & $13(23.2 \%)$ & $6(23.1 \%)$ & \\
\hline Soft tissue & $5(8.9 \%)$ & $2(7.7 \%)$ & \\
\hline Preoperative chemotherapy & & & 0.707 \\
\hline Yes & $32(57.1 \%)$ & $16(61.5 \%)$ & \\
\hline No & $24(42.9 \%)$ & $10(38.5 \%)$ & \\
\hline Postoperative chemotherapy & & & 0.080 \\
\hline Yes & $51(91.1 \%)$ & $20(76.9 \%)$ & \\
\hline No & $5(8.9 \%)$ & $6(23.1 \%)$ & \\
\hline Stem cell transplantation & & & 0.458 \\
\hline Yes & $15(26.8 \%)$ & $5(19.2 \%)$ & \\
\hline
\end{tabular}

Note: ${ }^{*} \mathrm{P}<0.05$ 


\begin{tabular}{|llll|}
\hline Characteristic & NLR冈3(n= 56) & NLR $\geq 3(n=26)$ & P Value \\
\hline No & $41(73.2 \%)$ & $21(80.8 \%)$ & \\
\hline Preoperative laboratory examination & & & \\
\hline Hb & $112.661 \pm 24.875$ & $100.577 \pm 25.906$ & $0.047^{\star}$ \\
\hline PLT & $208.375 \pm 77.386$ & $196.885 \pm 103.643$ & 0.577 \\
\hline ALb & $32.884 \pm 6.020$ & $31.158 \pm 6.287$ & 0.237 \\
\hline AST & $22.893 \pm 8.392$ & $33.962 \pm 18.790$ & $0.007 *$ \\
\hline ALT & $19.893 \pm 10.283$ & $28.462 \pm 24.173$ & 0.093 \\
\hline Г-GT & $34.107 \pm 27.520$ & $47.581 \pm 38.186$ & 0.073 \\
\hline Blood $\beta 2-M G$ & $5.430 \pm 9.076$ & $4.095 \pm 2.847$ & 0.475 \\
\hline Cr & $84.539 \pm 73.734$ & $89.377 \pm 85.843$ & 0.794 \\
\hline Note: ${ }^{*}$ P $<0.05$ & & & \\
\hline
\end{tabular}

Changes of peripheral blood absolute lymphocyte count and absolute neutrophil count before and after operation

The peripheral blood absolute lymphocyte count was at a low level at all stages before and after operation. In particular, that 1 week after operation $(1.186 \pm 0.548 \times 109 / \mathrm{L})$ declined, that 1 month after operation $(1.453 \pm 0.755 \times 109 / \mathrm{L})$ returned to that before operation, while that at the last follow-up visit $(1.147 \pm 0.824 \times 109 / \mathrm{L})$ declined again. The absolute neutrophil count 1 week after operation $(4.778 \pm$ $2.372 \times 109 / \mathrm{L})$ increased significantly, and that 1 month after operation $(3.724 \pm 1.961 \times 109 / \mathrm{L})$ returned to that before operation (Table 4) (Fig. 5). The peripheral blood NLR after operation (4.562 \pm 2.328$)$ increased significantly, and that 1 month after operation $(3.138 \pm 2.190)$ declined, while that at the last follow-up visit (5.032 \pm 6.726$)$ increased again (Table 4) (Fig. 6). 
Table 4

One-Way ANOVA Results of Peripheral Blood Absolute Lymphocyte Counts, Absolute Neutrophil Counts and Blood NLRs of the 82 MMBD Patients at Different Time Points

\begin{tabular}{|c|c|c|c|}
\hline Before operation & 1 week after operation & 1 month after operation & Last follow-up visitP \\
\hline $1.395 \pm 0.745$ & $1.186 \pm 0.548$ & $1.453 \pm 0.755$ & $1.147 \pm 0.824^{(1)(2)}$ \\
\hline $25.723 \pm 11.016$ & $19.329 \pm 9.083^{(3)(4)}$ & $26.658 \pm 10.699$ & $22.826 \pm 12.683$ \\
\hline $3.872 \pm 2.074$ & $4.778 \pm 2.372^{(5)(6)(7)}$ & $3.724 \pm 1.961$ & $3.364 \pm 2.042$ \\
\hline $2.783 \pm 1.966^{(8)(9)}$ & $4.562 \pm 2.328$ & $3.138 \pm 2.190^{(10)}$ & $5.032 \pm 6.726$ \\
\hline \multicolumn{4}{|c|}{$\begin{array}{l}\text { Note: (1) When that at the last follow-up visit was compared to that } 1 \text { week after operation, } P=0.055 \text {; } \\
\text { (2) When that at the last follow-up visit was compared to that } 1 \text { week after operation, } P<0.05 ;(3) \\
\text { When that } 1 \text { week after operation was compared to that before operation, } P<0.05 \text {; }(4) \text { When that } 1 \\
\text { week after operation was compared to that } 1 \text { month after operation, } P<0.05 ;(5) \text { When that } 1 \text { week } \\
\text { after operation was compared to that before operation, } P<0.05 ;(6) \text { When that } 1 \text { week after operation } \\
\text { was compared to that } 1 \text { month after operation, } P<0.05 ;(7) \text { When that } 1 \text { week after operation was } \\
\text { compared to that at the last follow-up visit, } P<0.05 ;(8) \text { When that before operation was compared to } \\
\text { that } 1 \text { week after operation, } P<0.05 ;(9) \text { When that before operation was compared to that at the last } \\
\text { follow-up visit, } P<0.05 ;(10) \text { When that } 1 \text { month after operation was compared to that at the last } \\
\text { follow-up visit, } P<0.05 \text {. }\end{array}$} \\
\hline
\end{tabular}

When analyzing preliminarily the immune status of the 82 MMBD patients before and after operation with the peripheral lymphocyte percentage as the evaluation indicator, the result showed that the peripheral lymphocyte percentage of MMBD patients before operation $(25.723 \pm 11.016 \%)$ was at a normal low level, that 1 week after operation $(19.329 \pm 9.083 \%)$ declined significantly, that 1 month after operation $(26.658 \pm 10.699 \%)$ returned to that before operation, and that at the last follow-up visit $(22.826 \pm 12.683 \%)$ returned to a low level again (Table 5) (Fig. 7).

Table 5

One-Way ANOVA Results of Peripheral Blood Lymphocyte Percentages at Different Time Points

$\begin{array}{llll}\text { Grouping } & \text { Mean } \pm \text { SD } & F & \text { P value }\end{array}$

Before operation

$25.723 \pm 11.016$

4.347

0.005

1 week after operation

$19.329 \pm 9.083^{*} \#$

1 month after operation

$26.658 \pm 10.699$

Last follow-up visit

$22.826 \pm 12.683$

Note: *When compared that 1 week after operation to that before operation, $\mathrm{P}<0.05$; \#When compared that 1 week after operation to that 1 month after operation, $\mathrm{P}<0.05$.

\section{Factors Affecting The Ps Of Mmbd Patients}


Multivariate survival analysis showed that postoperative chemotherapy $(P=0.000)$ and preoperative peripheral blood NLR $(P=0.015)$ were independent risk factors affecting POS (Table 6).

Table 6

Multivariate Cox Regression Analysis of Risk Factors Affecting the POS of MM Patients

\begin{tabular}{|llll|}
\hline Characteristic & P Value & HR & 95\% Cl \\
\hline Age & 0.202 & 1.023 & $0.988-1.058$ \\
\hline Gender & 0.083 & 0.574 & $0.306-1.075$ \\
\hline ISS stage & 0.396 & - & - \\
\hline Part & 0.878 & - & - \\
\hline Number of lesions & 0.186 & - & - \\
\hline Surgery Method & 0.170 & - & - \\
\hline Preoperative chemotherapy & 0.397 & 1.341 & $0.680-2.642$ \\
\hline Postoperative chemotherapy & $0.000 *$ & 7.492 & $2.753-20.389$ \\
\hline Preoperative peripheral blood NLR & $0.015 *$ & 1.209 & $1.038-1.408$ \\
\hline Note: ${ }^{*}<0.05$ & & & \\
\hline
\end{tabular}

\section{Discussion}

\section{Clinical value of peripheral blood NLR in predicting the prognosis of MMBD patients treated with operation}

Multiple myeloma is a malignant proliferative disease of plasma cells, whose occurrence and development are strongly dependent on the micro-environment. The interaction between MM cells and the micro-environment plays a crucial role in the pathogenesis of $\mathrm{MM}^{[10-12]}$. MM's micro-environment is mainly composed of inflammatory cells (including macrophages, dendritic cells, mast cells and myeloidderived suppressor cells), which are the main source of MM infiltrating bone marrow cytokines and mediate immune suppression of $\mathrm{MM}^{[13-16]}$. Inflammation is one of the characteristics of malignant tumors, and tumor-related inflammation plays an important role in promoting tumorigenesis by inducing tumor cell growth, angiogenesis and genomic instability ${ }^{[17]}$. Neutrophil-lymphocyte ratio is a readily available indicator of systemic inflammatory reaction ${ }^{[18]}$. As a simple and inexpensive tool, NLR has been more and more reported to have predictive and prognostic value in solid tumors, such as gastric cancer, liver cancer and ovarian cancer. Elevated NLR indicates poor prognosis in patients, but its mechanism is not yet clear. And there are few studies on hematologic tumors ${ }^{[19-23]}$. NLR has been reported to be of great value in the prognosis of MM patients. Studies have shown that elevated NLR is associated with declined OS in MM patients receiving bortezomib induction therapy ${ }^{[24]}$. Studies have also shown that 
elevated NLR and declined platelet-lymphocyte ratio (PLR) are independent prognostic factors for progression-free survival of MM patients ${ }^{[25]}$. Therefore, elevated NLR and declined monocyte-lymphocyte ratio (MLR) and PLR predict adverse clinical outcomes in MM patients, and may serve as cost-effective and readily available prognostic biomarkers ${ }^{[26]}$. So far, there are no reports on the prognostic value of NLR in MM patients treated with operation. Results in the study showed that the NLR cut-off values of the preoperative peripheral blood NLR $\geq 3$ group and the preoperative peripheral blood NLR $\geq 4$ group were significantly correlated with the POS and postoperative cumulative survival rate. In particular, when the NLR cut-off value was set as $\geq 3$, the difference was the most significant. Compared with patients in the low NLR group, those in the high NLR group had statistical differences in the number of lesions, preoperative hemoglobin level, preoperative glutamate transaminase, and preoperative absolute lymphocyte count. Multivariate survival analysis showed that postoperative chemotherapy and preoperative peripheral blood NLR were independent risk factors affecting the PS of patients.

\section{Change laws of peripheral blood NLR before and after operation}

Due to the great range of removal of tumor tissues in operations, the tumor load decreased significantly, and the stimulating effect of tumor cells on the immune and inflammatory reaction was reduced, resulting in great changes of the NLR before and after operation. Researchers in NLR changes in patients that underwent radical resection of non-small cell lung cancer before and after operation found that the NLR of a patient 1 month after operation increased by over 0.27 , and the DFS and OS of the same patient were significantly shorter than those whose NLR declined or increased by less than 0.27 . They believed that the change of NLR from that before operation to that 1 month after operation could be used for the early evaluation of the effectiveness of tumor therapy ${ }^{[27]}$.

It was found in the study that MM patients had a high NLR before operation, which increased significantly 1 week after operation. In particular, in the second week after operation, the absolute neutrophil count increased significantly while the absolute lymphocyte count declined, in the second month after operation, the NLR declined while the absolute neutrophil count and absolute lymphocyte count returned to those before operation, and at the last follow-up visit, the NLR returned to a high level while the absolute neutrophil count increased significantly and the absolute lymphocyte count declined significantly. Such change laws indicate that a high preoperative NLR suggests a poor prognosis in MM patients, which may be the result of the imbalance of inflammatory reaction between anti-tumor and protumor effects ${ }^{[26]}$. An elevated NLR indicates that the neutrophils in an MM patient's blood increased while the lymphocytes decreased, resulting in an imbalance between the anti-tumor effect of lymphocytes and the pro-tumor effect of neutrophils, thus affecting the prognosis of tumor patients.

\section{Changes of the immune status of MMBD patients before and after operation and its effect on prognosis}

Cellular immunity and humoral immunity play a key role in the occurrence and progression of tumors, and the function and status of cellular immunity largely reflect the progress of tumors and the prognosis of patients. At present, the clinical application of anti-tumor cell immunotherapy based on the correlation 
between cellular immunologic function and status and tumors is being more and more applied to clinical practice. Correct judgment and evaluation of the cellular immunologic function of patients with malignant tumor play an important role in evaluating the development of disease and guiding clinical treatment. Multiple myeloma is characterized by immunologic dysfunction Recently, MM's immune injury has been considered as another tool to predict prognosis ${ }^{[27]}$. Long-term survival in MM patients may be the result of unique immunologic characteristics, the most important of which is the reduction of immunosuppression ${ }^{[28]}$. Lymphocytes are an important part of the body's immune system. They contain subsets of different functions, which can be roughly divided into T cells, B cells and NK cells. With the continuous deepening of studies on the cellular and humoral immune functions in MM patients, domestic and foreign studies have found that there are various defects in the immune system of MM patients, including quantity anomaly and functional defect of B cells, T cells, NK cells and DC cells, as well as abnormal regulatory Tregs ${ }^{[29-30]}$.

NK cells are derived from hematopoietic stem cells, which have the characteristics of MHC non-restriction and kill tumor cells without being activated. Bernal et al. ${ }^{[31]}$ found in their study that the number of NK cells in peripheral blood of MM patients increased while their activity declined. Jurisic et al. ${ }^{[32]}$ proved in their study that the decline of NK cell activity in patients was related to disease progression and the degree of plasma cell infiltration.

T cells play a major role in the anti-tumor immune reaction of tumor patients, and regulate the immune reaction of body and maintain immune stability. CD4/CD8 ratio reflects the cellular immunologic function and status of the body. In particular, CD4 + T cells are mainly helper T cells (Th), being the main reaction cells in the immune reaction, and CD8 $+T$ cells are mainly composed of cytotoxic $T$ cells and inhibitory $T$ cells, producing cytotoxic effects on target cells. T cell subset expression anomaly plays an important role in the pathogenesis of MM. Koike and Oken et al. have found in their studies that there is a decrease in $\mathrm{CD} 4+\mathrm{T} / \mathrm{CD} 8+\mathrm{T}$ in MM patients, which breaks the immune balance of MM patients and weakens their existing anti-tumor functions ${ }^{[33-34]}$. Treg is a subset of T cells that control autoimmunity and have negative immune regulation function, which maintains the immune system stability of body together with Th1 and Th2. It is related to the immune tolerance and tumor immune escape mechanism ${ }^{[35-36]}$. In recent years, studies by domestic and foreign scholars have shown that the proportion of Th1 and Th2 in MM patients is lower than that in patients in the healthy control group, suggesting that both humoral and cellular immunity of MM patients are damaged to varying degrees ${ }^{[37]}$. Therefore, lymphocyte subsets and Th cells are of great significance in the prognostic evaluation of MM patients. Lymphocytes play a key role in cell-mediated anti-tumor immune reaction, and their amount reflects the degree of reaction of the host immune system.

Whether operations affect the immune status of MM patients has not been reported. Results of the paper showed that the lymphocyte percentage of MM patients was at a normal low level before operation, which declined significantly 1 week after operation, returned to that before operation 1 month after operation, and returned to a low level at the last follow-up visit. They suggest that operations did affect 
the immune status of MM patients. After the operation, the immune balance of MM patients was broken in a short period of time, making them in low immune status. However, as time went by, their immune status recovered spontaneously, and returned to that before operation about 1 month after operation. Therefore, for MM patients that underwent operations, adjuvant therapy to enhance their immunity may be given when necessary, so as to allow them to be in a sound immune status, which may be helpful to the overall treatment of MM patients. The correlation between such results and the timing of chemotherapy after operation needs further study.

Neutrophils play a multivariate role in tumor diseases, but its role in cancer has not been fully understood $^{[38]}$. Neutrophils reflect the inflammatory state of tumor patients and play different roles in different stages of tumor diseases ${ }^{[39]}$. Neutrophils release reactive nitrogen species (RNS), reactive oxygen species (ROS) or proteases that promote the occurrence of tumors ${ }^{[40]}$. The nitric-oxide synthase (iNOS) or argininase1 (ARG1) released by neutrophils inhibits the anti-tumor reaction of CD8 + T lymphocytes ${ }^{[41]}$ by weakening the immune system to promote tumor proliferation and stimulated by TGF$\beta$, and promotes metastasis and diffusion by inhibiting the natural killing function and promoting tumor cell extravasation ${ }^{[42]}$. In view of the role of neutrophils in the occurrence and development of tumor diseases, some researchers believed that the increase of neutrophil count was one of the adverse prognostic factors of head and neck cancer ${ }^{[43]}$.

Results of the paper showed that the neutrophils of MM patients were at a normal level before operation, which increased significantly 1 week after operation, returned to that before operation 1 month after operation, and was lower than that before operation at the last follow-up visit. In our opinion, patients were in the postoperative inflammatory reaction period 1 week after operation, so their neutrophils were at a high level at that time. However, as time went by, their immune status after operation recovered, and returned to that before operation about 1 month after operation. This may be a normal body reaction, but whether such reaction affects tumors needs further study. At the last follow-up visit, the tumor status of patients was improved and the level of their neutrophils declined over that before operation due to the application of treatment measures such as operations and chemotherapy.

To sum up, changes of peripheral blood NLR were strongly correlated to the changes of lymphocyte count and neutrophil count and, during an operation, the peripheral blood NLR increased while the neutrophil count elevated and the lymphocyte count declined. Such a law of elevation may be a normal reaction of the body and not clearly correlated with the prognosis of tumors. Peripheral blood NLR before and 1 month after operation may reflect the immune status of patients from one side, and can be used as one of the indicators to predict operative prognoses.

To some extent, the study was inadequate. Firstly, some of the $82 \mathrm{MM}$ patients recruited for the study lacked the lymphocyte subset information at different time points due to the large time span of diagnosis and treatment and varying locations of follow-up visits. Secondly, there might be some errors in the results of the study due to different treatment regimens for patients during the treatment period. Thirdly, the detection of peripheral blood-related cells alone failed to fully reflect the immune status of body. So, 
the specific mechanism of MM-induced changes in the immune status of body still needs further study and analysis. Among the patients that underwent operations, the number of patients undergoing operations at different sites and by different means were limited, so the multivariate test was unfeasible, while the results of the univariate test did have errors.

\section{Conclusion}

Preoperative peripheral blood NLR was significant for suggesting the prognosis of MMBD treated with operations, while MMBD patients with high NLR before operation have a poor prognosis. The immune status of MMBD patients was at a normal low level, and significantly declined after operation and returned to that before operation 1 month after operation. Inflammation and immunity may simultaneously affect the micro-environment and occurrence and development of MM tumors.

\section{Abbreviations}

\section{NLR}

Neutrophil-lymphocyte ratio

\section{MM}

Multiple myeloma

MMBD

Multiple myeloma bone disease

CT

Computed tomography

MRI

Magnetic resonance imaging

DS

Durie/Salmon

ISS

International staging system

POS

Postoperative survival

\section{Declarations}

\section{Ethical approval and consent to participate}

Beijing Chao-yang Hospital Human Research Ethics Committee classifies this as a retrospective audit and, as such, does not require ethical submission/approval or consent to participate.

\section{Consent for publication}


Beijing Chao-yang Hospital Human Research Ethics Committee classifies this as a retrospective audit and, as such, does not require consent to publish.

\section{Availability of data and materials}

The datasets collected during and/or analyzed during the current study are available from the corresponding author upon reasonable request.

\section{Competing interests}

The authors declare that they have no competing interests.

\section{Funding}

This study was supported by the grants from the Beijing Municipal Natural Science Foundation(no. 7192071).

\section{Authors' contributions}

$\mathrm{ZYX}$ is the primary author and contributed in drafting and revising the manuscript. ZYX contributed to the data collection and drafting of the manuscript. XCY/XJS contributed to the data analysis and interpretation. XRD/ZYX contributed to the conception of the article and manuscript revision. All authors read and approved the final manuscript.

Acknowledgments We wish to thank Dr. Aijun-Liu and Dr. Wenming-Chen of the Beijing Chao-Yang Hospital Hematology Dept for their help in the statistical analysis and data processing.

\section{References}

1. Rajkumar SV. Multiple myeloma: 2016 update on diagnosis, risk-stratification, and management [J]. Am J Hematol. 2016;91(7):719-34.

2. Miller JA, Balagamwala EH, Chao ST, et al. Spine stereotactic radiosurgery for the treatment of multiple myeloma [J]. J Neurosurg Spine. 2017;26(3):282-90.

3. Surgeon's Committee of the Chinese Myeloma Working Group of the International Myeloma F. Consensus on Surgical Management of Myeloma Bone Disease [J]. Orthop Surg. 2016;8(3):263-9.

4. Yao X, Xu Z, Du X. PKP/PVP combine chemotherapy in the treatment of multiple myeloma patients with vertebral pathological fractures: minimum 3-year follow-up of 108 cases [J]. Journal of Orthopaedic Surgery and Research, 2019, 14(1).

5. Diem S, Schmid S, Krapf M, et al. Neutrophil-to-Lymphocyte ratio (NLR) and Platelet-to-Lymphocyte ratio (PLR) as prognostic markers in patients with non-small cell lung cancer (NSCLC) treated with nivolumab [J]. Lung Cancer. 2017;111(24):176-81. 
6. Sun S, Wang X, Chen J. Using Pre-Treatment Neutrophil-to-Lymphocyte Ratio to Predict the Prognosis of Young Patients with Hepatocellular Carcinoma Implemented Minimally Invasive Treatment [J].J Adolesc Young Adult Oncol, 2019,17.

7. Luvira V, Kamsa-Ard S, Pugkhem A, et al. Predictive utility of neutrophil-to-lymphocyte ratio and platelet-to-lymphocyte ratio in intraductal papillary neoplasm of the bile duct [J]. Clin Exp Hepatol. 2019;5(3):250-5.

8. Zeng Q, Liu Z, Li Q, et al. Prognostic value of neutrophil to lymphocyte ratio and clinicopathological characteristics for multiple myeloma: A meta-analysis [J]. Med (Baltim). 2018;97(41):e12678.

9. Liu S, Shi J, Guo H, Xu F, Wei M, Sun K, et al. Prognostic Significance Of The Inflammatory IndexBased Scoring System In Patients Preliminarily Diagnosed With Multiple Myeloma In The Bortezomib-Based Chemotherapy Era [J]. Cancer Manag Res. 2019;11:9409-20.

10. Kumar S. Multiple myeloma-current issues and controversies [J]. Cancer Treat Rev. 2010;36(Suppl 2):3-11.

11. Spicka I. Advances in multiple myeloma therapy during two past decades [J]. Comput Struct Biotechnol J. 2014;10:38-40.

12. Palumbo A, Anderson K. Multiple myeloma [J]. N Engl J Med. 2011;364:1046-60.

13. Prabhala RH, Pelluru D, Fulciniti M, et al. Elevated IL-17 produced by TH17 cells promotes myeloma cell growth and inhibits immune function in multiple myeloma [J]. Blood. 2010;115:5385-92.

14. Chauhan D, Singh AV, Brahmandam M, et al. Functional interaction of plasmacytoid dendritic cells with multiple myeloma cells: a therapeutic target [J]. Cancer Cell. 2009;16:309-23.

15. Tai YT, Anderson KC. B cell maturation antigen (BCMA)-based immunotherapy for multiple myeloma [J]. Expert Opin Ther. 2019;19(11):1143-56.

16. Gorgun GT, Whitehill G, Anderson JL, et al. Tumor-promoting immune-suppressive myeloid-derived suppressor cells in the multiple myeloma microenvironment in humans [J]. Blood. 2013;121:297587.

17. Zheng Y, Cai Z, Wang S, et al. Macrophages are an abundant component of myeloma microenvironment and protect myeloma cells from chemotherapy drug-induced apoptosis [J]. Blood. 2009;114(17):3625-8.

18. Cummings $M$, Merone $L$, Keeble $C$, et al. Preoperative neutrophil:lymphocyte and platelet:lymphocyte ratios predict endometrial cancer survival [J]. British journal of cancer. 2015;113(2):311-20.

19. Luo G, Guo M, Liu Z, et al. Blood neutrophil-lymphocyte ratio predicts survival in patients with advanced pancreatic cancer treated with chemotherapy [J]. Ann Surg Oncol. 2015;22(2):670-6.

20. Guthrie GJ, Charles KA, Roxburgh CS, et al. The systemic inflammation-based neutrophil lymphocyte ratio: Experience in patients with cancer [J]. Crit Rev Oncol Hematol. 2013;88(1):218-30.

21. Feng JF, Liu JS, Huang Y. Lymphopenia predicts poor prognosis in patients with esophageal squamous cell carcinoma [J]. Med (Baltim). 2014;93(27):e257. 
22. Kokcu A, Kurtoglu E, Celik H, et al. May the platelet to lymphocyte ratio be a prognostic factor for epithelial ovarian cancer [J]. Asian Pac J Cancer Prev. 2014;15(22):9781-4.

23. Gungorduk K, Ertas IE, Ozdemir A, et al. Prognostic Significance of Retroperitoneal Lymphadenectomy, Preoperative Neutrophil Lymphocyte Ratio and Platelet Lymphocyte Ratio in Primary Fallopian Tube Carcinoma: A Multicenter Study [J]. Cancer Res Treat. 2015;47(3):480-8.

24. Zhou X, Wang J, Xia J, et al. Evaluation of neutrophil-to-lymphocyte ratio in newly diagnosed patients receiving bortezomib-based therapy for multiple myeloma [J]. Cancer Biomark. 2018;22(1):43-8.

25. Wongrakpanich S, George G, Chaiwatcharayut W, et al. The prognostic significance of neutrophil-tolymphocyte and platelet-to-lymphocyte ratios in patients with multiple myeloma [J]. J Clin Lab Anal. 2016;30:1208-13.

26. Shi L, Qin X, Wang H, et al. Elevated neutrophil-to-lymphocyte ratio and monocyte-to-lymphocyte ratio and decreased platelet-to-lymphocyte ratio are associated with poor prognosis in multiple myeloma [J]. Oncotarget. 2017;8(12):18792-801.

27. Pessoa de Magalhaes RJ, Vidriales MB, Paiva B, et al. Analysis of the immune system of multiple myeloma patients achieving long-term disease control by multidimensional flow cytometery [J]. Haematologica. 2013;98(1):79-86.

28. Bryant $\mathrm{C}$, Suen $\mathrm{H}$, Brown $\mathrm{R}$, et al. Long-term survival in multiple myeloma is associated with a distinct immunological profile, which includes proliferative cytotoxic T-cell clones and a favourableTreg/Th17 balance [J]. Blood Cancer J. 2013;3:e148.

29. Feyler S, von Lilienfeld-Toal M, et al. CD4(+) CD25(+) FoxP3(+) regulatory T cells are increased whilst CD3(+) CD4(-) CD8(-) alphabeta TCR(+) Double Negative T cells are decreased in the peripheral blood of patients with multiple myeloma which correlates with disease burden [ $\mathrm{J}$. $\mathrm{Br} \mathrm{J}$ Haematol. 2009;144(5):686-95.

30. Pratt G, Goodyear O, Moss P. Immunodeficiency and immunotherapy in multiple myeloma [J]. Br J Haematol. 2007;138(5):563.

31. Bernal M, Garrido P, Jimenez $P$, et al. Changes in activatory and inhibitory natural killer(NK) receptors may induce progression to multiple myeloma: implications for tumor evasion of T and NK cells [J]. Hum Immunol. 2009;70(10):854-7.

32. Jurisic V, Srdric T, Konjevic G, et al. Clinical stage-depanding decrease of NK cell activity in multiple myeloma patients [J]. Med Oncal. 2007;24(3):312-7.

33. Koike M, Sekigawa I, Okada M, et al. Relationship between CD4 (+)/CD8(+) T cell ratio and T cell activation in mulliple myeloma: reference to IL-16 [J]. Leuk Res. 2002;26(8):705-11.

34. Oken MM, Kay NE. T-cell subpopulations in multiple myeloma: correlation with clinical diseases status [J]. Br J Haematol. 1981;49(4):629-34.

35. Tu JF, Ding YH, Ying XH, et al. Regulatory T cells, especially ICOS + FOXP3 + regulatory T cells, are increased in the hepatocellular carcinoma microenvironment and predict reduced survival [J]. Sci Rep. 2016;11(6):35-6.

36. Fan L, Strasser-Weippl K, Li JJ, et al. Breast cancer in China [J]. Lancet Oncol. 2014;15(7):e279-89. 
37. Beyer M, Kochanek M, Giese T, et al. In vivo peripheral expansion of native CD4 + CD25high FOXP3 + regular T cells in patients with multiple myeloma [J]. Blood. 2006;107(10):3940-9.

38. Grecian R, Whyte MKB, Walmsley SR. The role of neutrophils in cancer [. J]Br Med Bull. 2018;128(1):5-14.

39. Coffelt SB, Wellenstein MD, de Visser KE. Neutrophils in cancer: neutral no more [J]. Nat Rev Cancer,2016,16:431-446.

40. Jamieson $T$, Clarke $M$, Steele $C W$, et al. Inhibition of CXCR2 profoundly suppresses inflammationdriven and spontaneous tumorigenesis [J]. J Clin Invest. 2012;122:3127-44.

41. Bodogai M, Moritoh K, Lee-Chang C, et al. Immunosuppressive and Prometastatic functions of myeloid-derived suppressive cells rely upon education from tumor-associated B cells [J]. Cancer Res. 2015;75:3456-65.

42. Spiegel A, Brooks MW, Houshyar S, et al. Neutrophils suppress Intraluminal NK cell-mediated tumor cell clearance and enhance Extravasation of disseminated carcinoma cells [J]. Cancer Discov. 2016;6:630-49.

43. Valero C, Pardo L, López M,et al. Pretreatment count of peripheral neutrophils, monocytes, and lymphocytes as independent prognostic factor in patients with head and neck cancer [. J]Head Neck. 2017;39(2):219-26.

\section{Figures}




\section{Survival of Two groups}

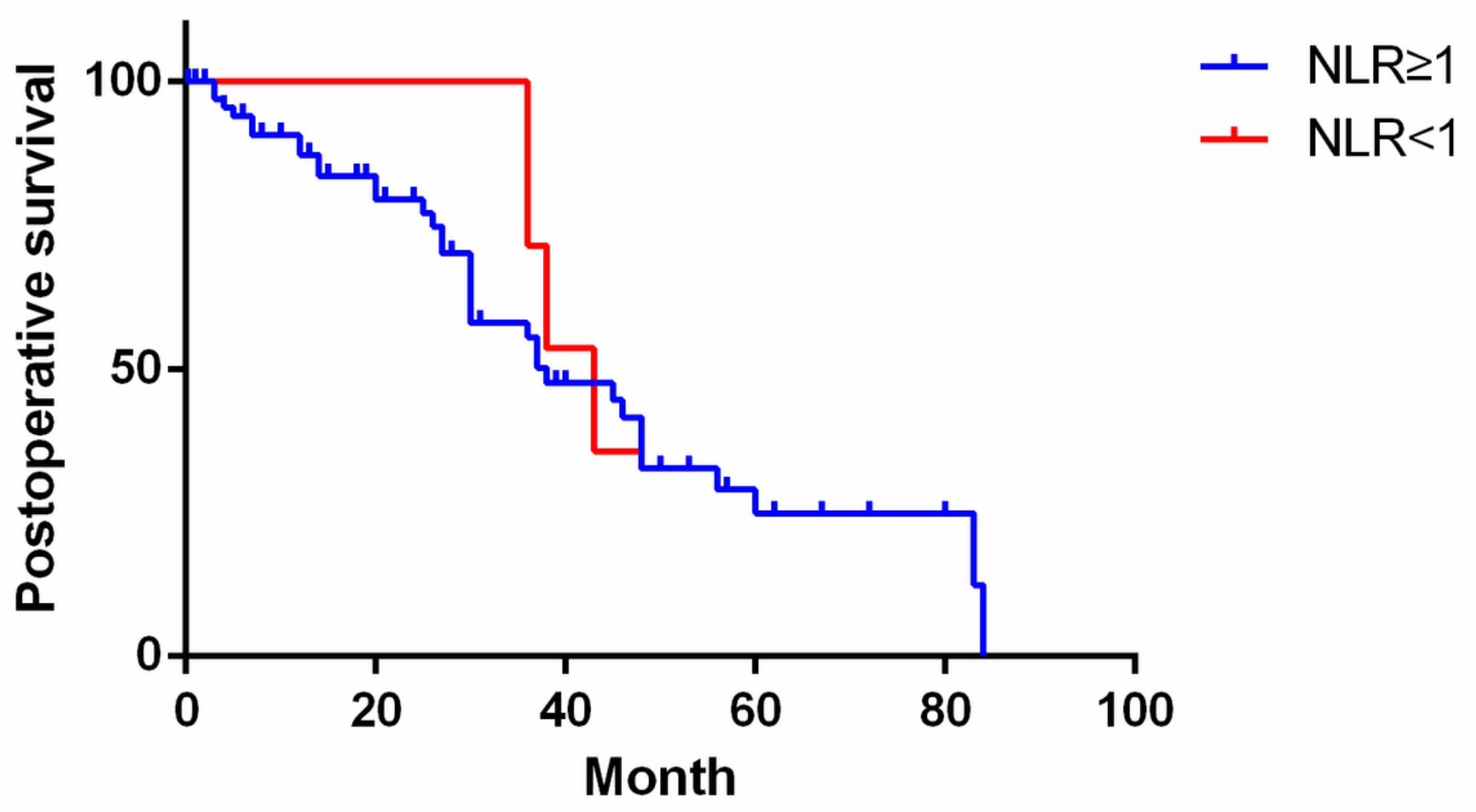

Figure 1

The POS of patients in the preoperative peripheral blood NLR $\geq 1$ group and the NLR $<1$ group had no significant statistical difference, $p>0.05$. 


\section{Survival of Two groups}

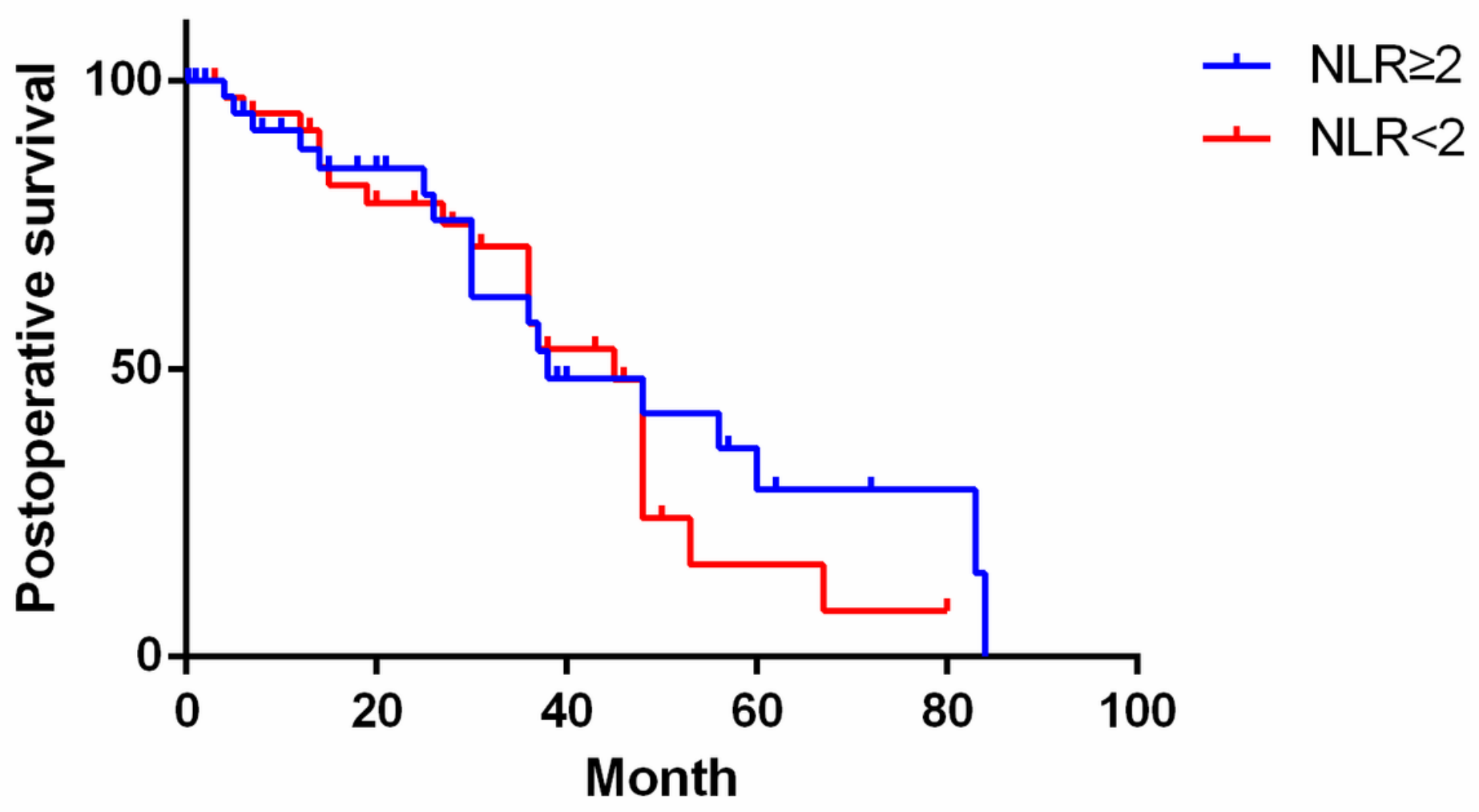

Figure 2

The POS of patients in the preoperative peripheral blood NLR $\geq 2$ group and the NLR $<2$ group had no significant statistical difference, $p>0.05$. 


\section{Survival of Two groups}

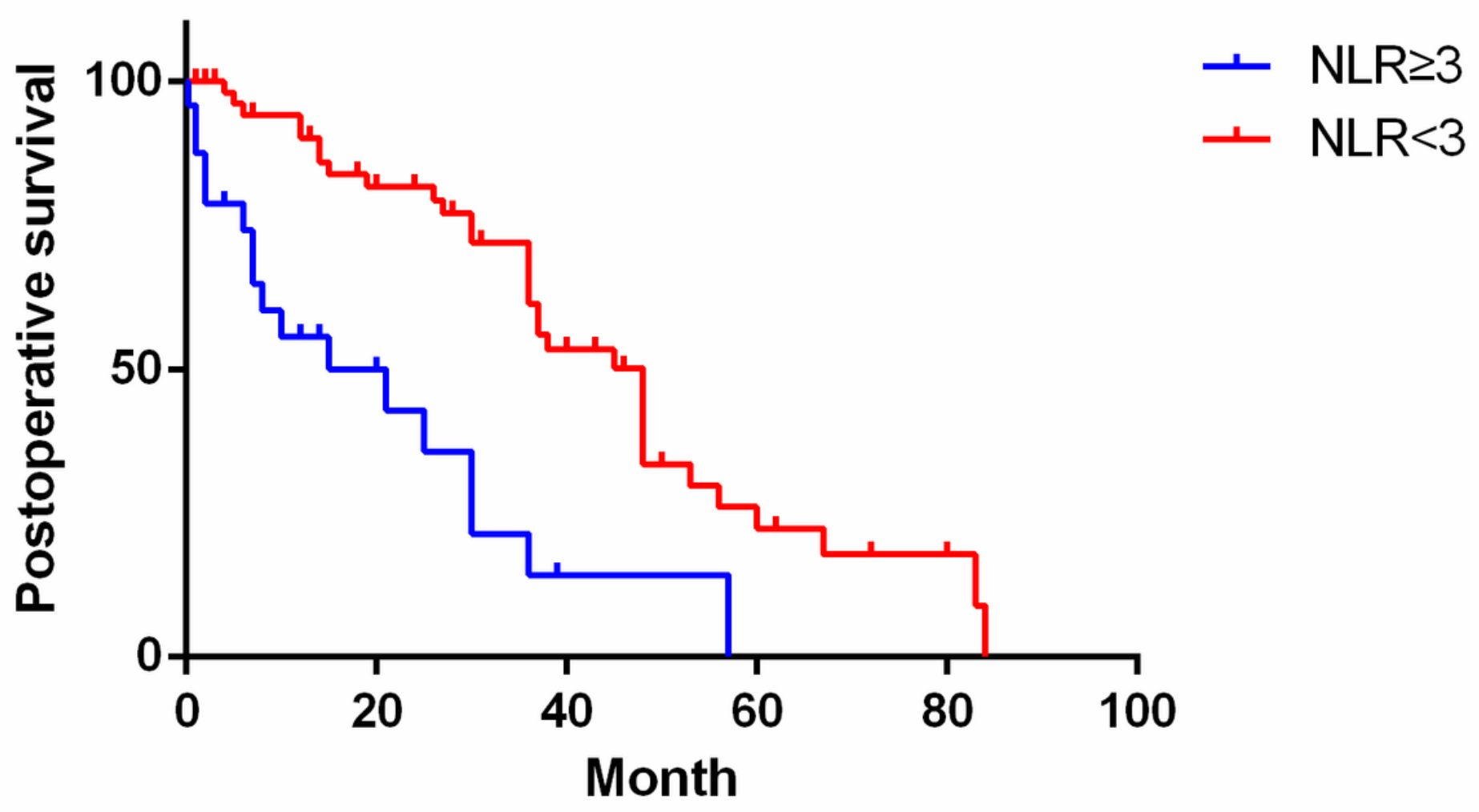

Figure 3

The POS of patients in the preoperative peripheral blood NLR $<3$ group was significantly longer than that in the NLR $\geq 3$ group, $p<0.05$. 


\section{Survival of Two groups}

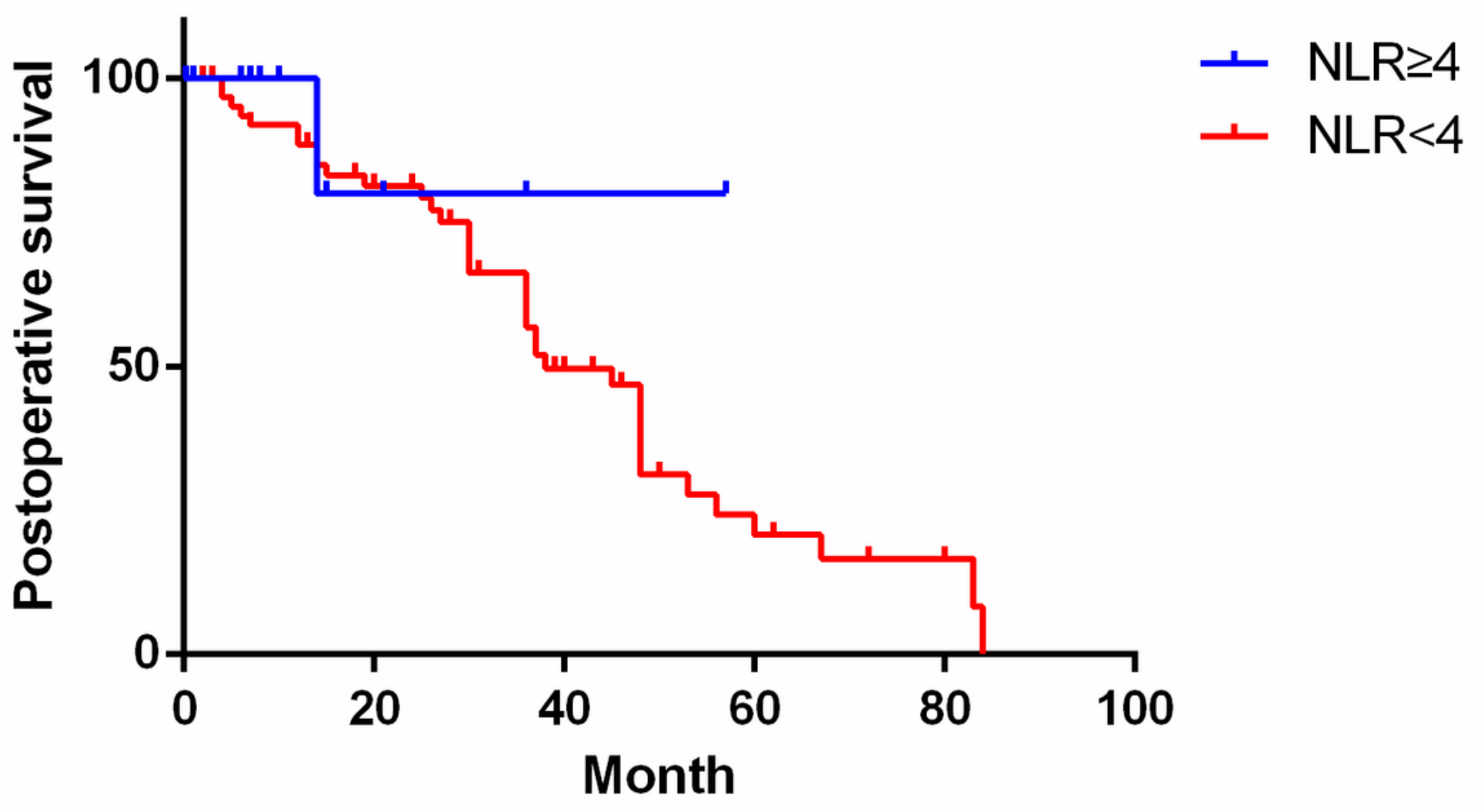

Figure 4

The POS of patients in the preoperative peripheral blood NLR $<4$ group was significantly longer than that in the NLR $\geq 4$ group, $p<0.05$. 

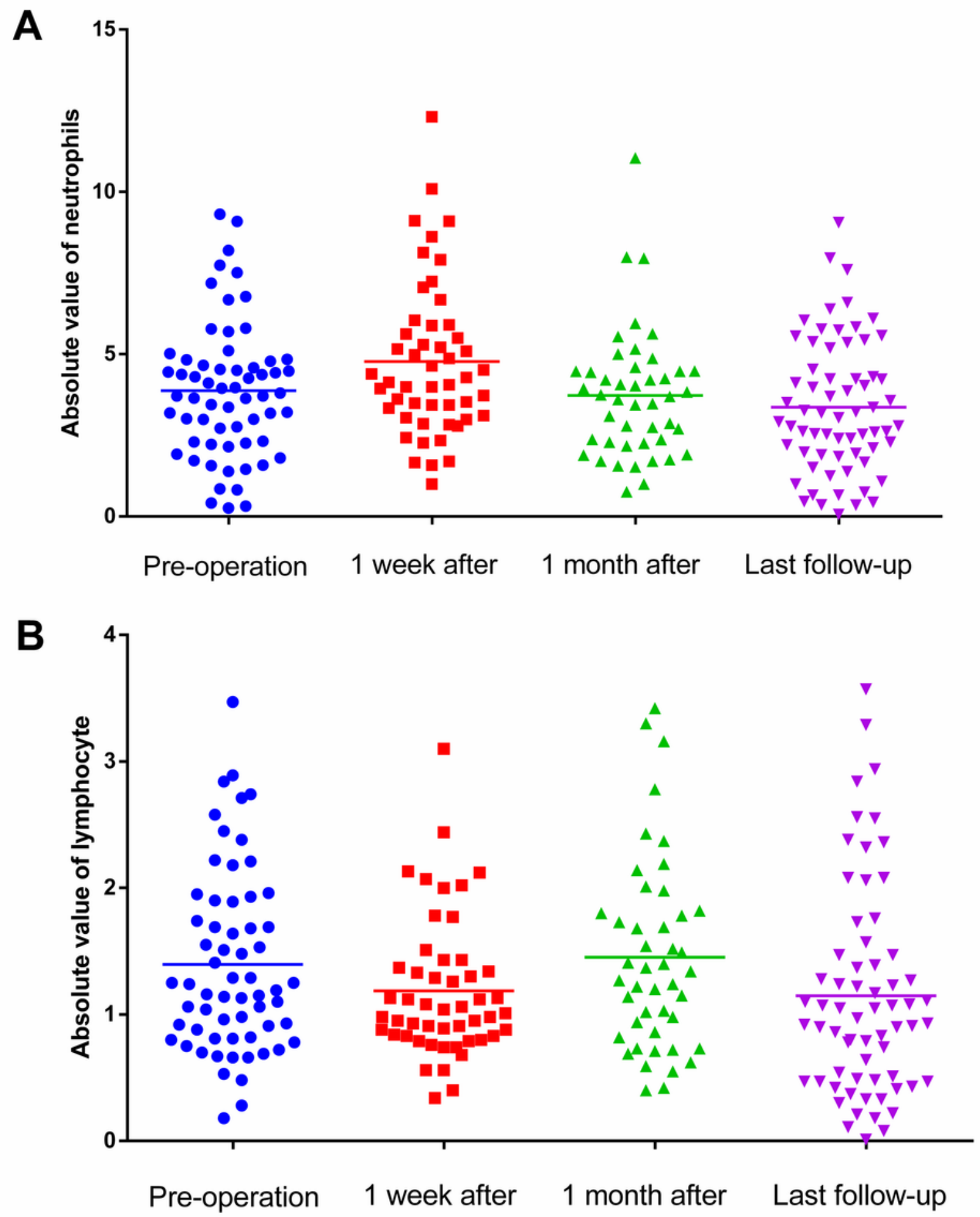

Figure 5

Peripheral blood absolute neutrophil count and absolute lymphocyte count of MMBD patients before and after operation The peripheral blood absolute lymphocyte count declined after operation, returned to that before operation 1 month after operation, and returned to a low level at the last follow-up visit. The absolute neutrophil count increased significantly 1 week after operation and returned to that before operation 1 month after operation. 

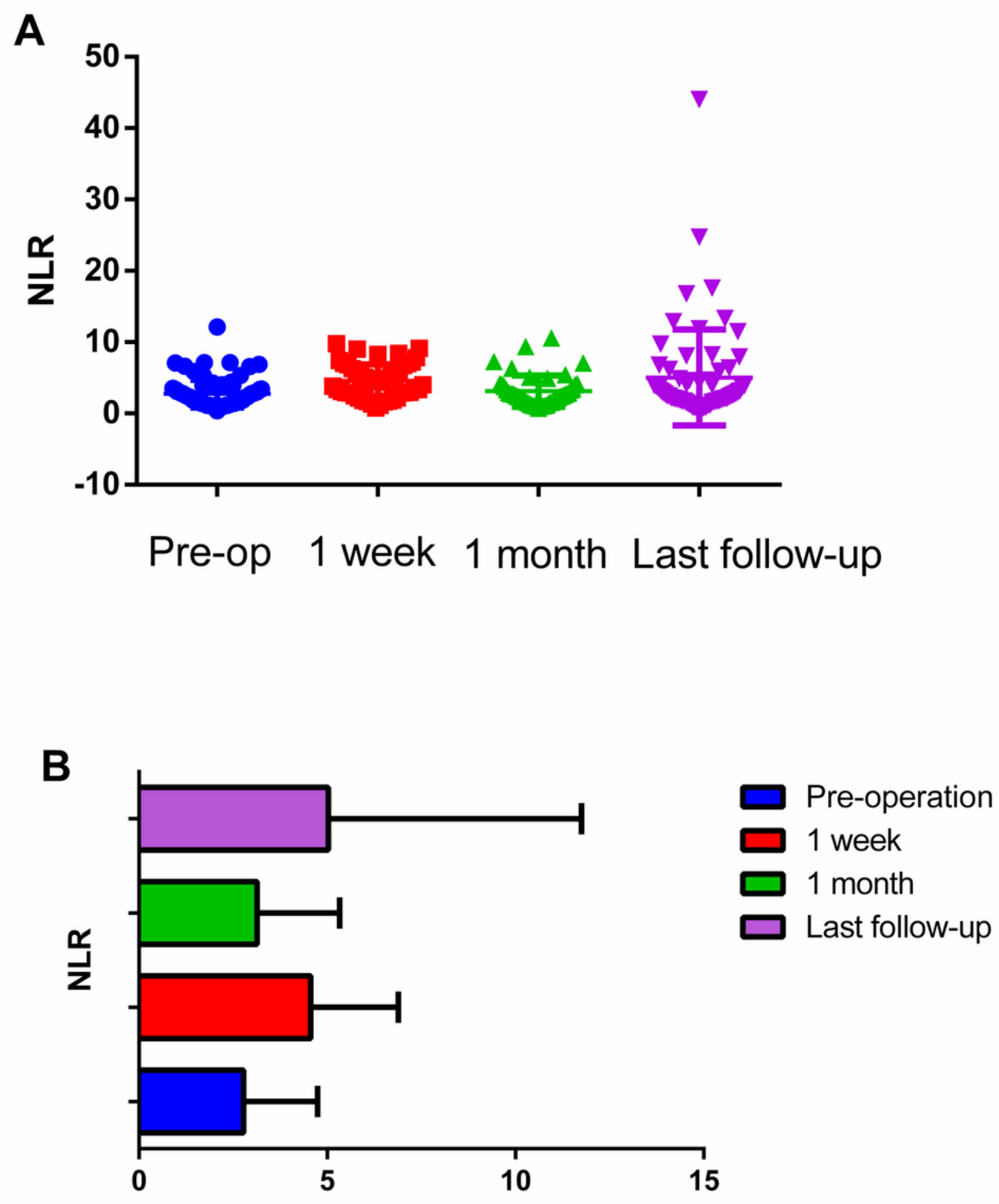

Figure 6

Change trend of peripheral blood NLR of MMBD patients before and after operation. The peripheral blood NLR increased significantly after operation, and declined 1 month after operation, while returning to a high level at the last follow-up visit. 


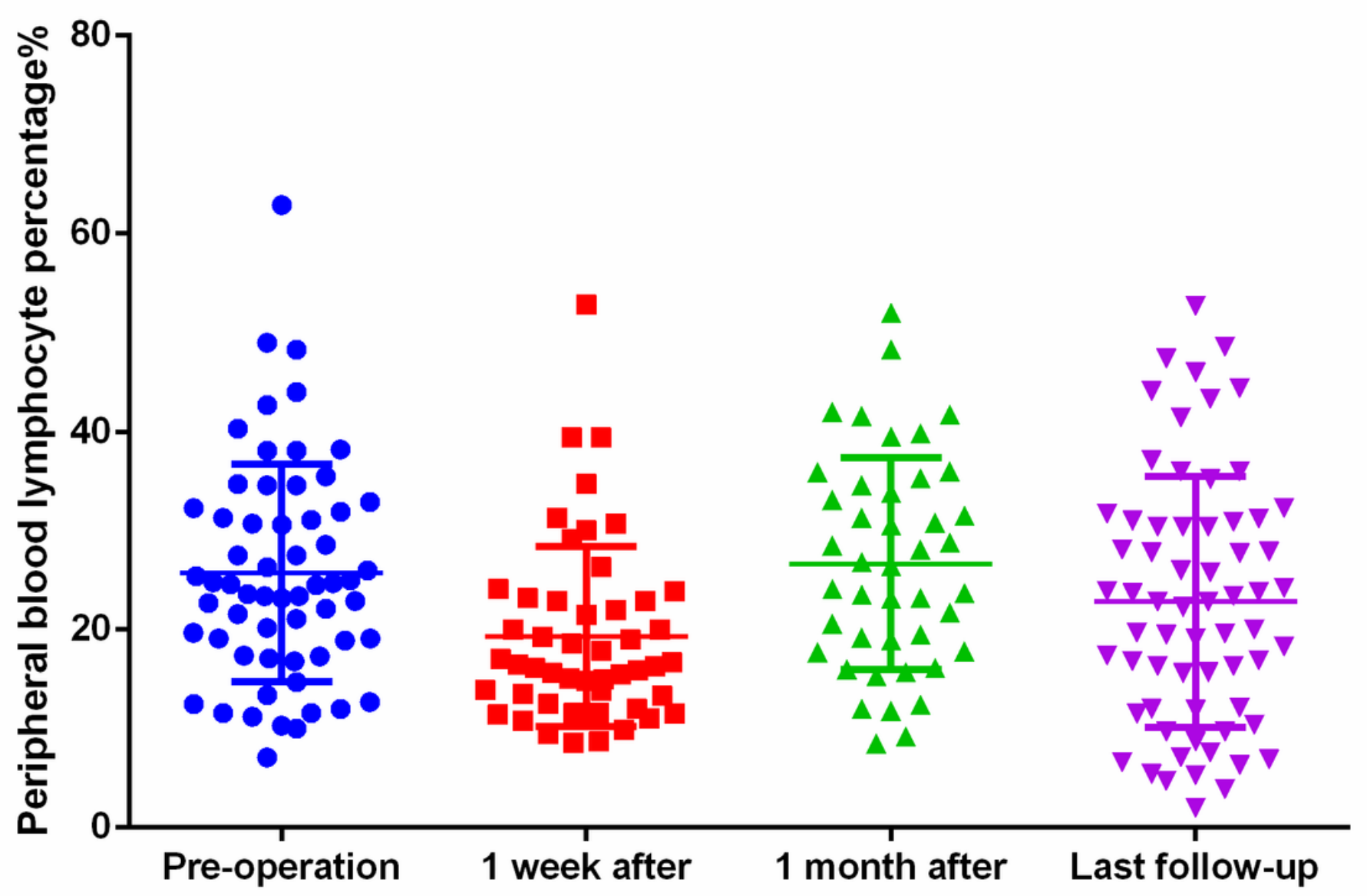

Figure 7

Peripheral blood lymphocyte percentage of MMBD patients before and after operation. The lymphocyte percentage of MMBD patients was at a normal low level and declined significantly 1 week after operation, while returning to that before operation 1 month after operation and returning to a low level at the last follow-up visit. 